\title{
Skin melanocytes: biology and development
}

\author{
Mirosława Cichorek, Małgorzata Wachulska, Aneta Stasiewicz, Agata Tymińska
}

Department of Embryology, Medical University of Gdansk, Poland

Head: Mirosława Cichorek PhD

Postep Derm Alergol 2013; XXX, 1: 30-41

DOI: $10.5114 /$ pdia.2013.33376

\begin{abstract}
In the human skin, melanocytes are present in the epidermis and hair follicles. The basic features of these cells are the ability to melanin production and the origin from neural crest cells. This last element is important because there are other cells able to produce melanin but of different embryonic origin (pigmented epithelium of retina, some neurons, adipocytes). The life cycle of melanocyte consists of several steps including differentiation of melanocyte lineage/s from neural crest, migration and proliferation of melanoblasts, differentiation of melanoblasts into melanocytes, proliferation and maturation of melanocytes at the target places (activity of melanogenic enzymes, melanosome formation and transport to keratinocytes) and eventual cell death (hair melanocytes). Melanocytes of the epidermis and hair are cells sharing some common features but in general they form biologically different populations living in unique niches of the skin.
\end{abstract}

Key words: melanocytes, neural crest cells, stem cells.

\section{Introduction}

Melanocytes form a heterogeneous group of cells in the human body. Although all of them have ability to produce melanin and originate from embryonic cells named neural crest cells (NCC), their particular functions in all target places are much wider than the melanin synthesis only [1]. In the human body melanocytes' presence does not confirm only epidermis, hair and iris where they give a color of these structures. Melanocytes have been also found in the inner ear, nervous system, heart and probably it is not the end of a list where these cells exist [2, 3]. It is necessary to stress that not only melanocytes have ability to produce melanin but also other cells e.g. cells of pigmented epithelium of retina, epithelia of iris and ciliary body of the eye, some neurons, adipocytes [4, 5].

The life cycle of melanocytes consists of several steps including lineage specification from embryonic neural crest cells (melanoblasts), migration and proliferation of melanoblasts, differentiation of melanoblasts into melanocytes, maturation of melanocytes (melanin production in special organelles - melanosomes, dendritic morphology), transport of mature melanosomes to keratinocytes and eventual cell death. Several populations of neural crest cells (cranial, dorsal trunk, ventral trunk) give melanocytes of the skin. The embryonic origin of epidermal and hair melanocytes is the same but development is different $[6,7]$. The problem of melanocyte stem cells' localization in the adult skin is still a matter of debate. The first such place was a hair bulge, but if only...? [8]. Experimental data clearly show that the trunk NCC migrating through a ventral pathway could remain in a myelin sheath of the cutaneous nerves and in particular situations give melanoblasts $[9,10]$. The embryonic development of melanocytes give an opportunity to better understand the skin diseases e.g. melanoma and its heterogeneity, vitiligo. Thus, in this review the epidermal and hair melanocytes' biology and development are characterized.

\section{Melanocyte in the skin as the epidermal melanin unit element}

Melanocytes molecularly are recognizable by identification of melanocyte-specific proteins as tyrosinase (TYR), tyrosinase-related protein 1 and 2 (TYRP1, TYRP2/DCT), melanosomal matrix proteins (Pmel17, MART-1), microphthalmia transcription factor (MITF) [1]. The microscopic analysis indicates that mature melanocytes are oval or fusiform, dendritic cells, smaller than keratinocytes. In the

Address for correspondence: Mirosława Cichorek PhD, Department of Embryology, Medical University of Gdansk, 1 Dębinki Str., 80-211 Gdansk, Poland, phone: +48 5834914 95, fax: +48 5834914 95, e-mail: cichorek@gumed.edu.pl

Received: 17.07.2012, accepted: 24.10.2012. 
cytoplasm there are present special membrane-bound organelles producing melanin, melanosomes [11]. Melanocytes reside in the basal layer of epidermis where they form the epidermal melanin units as a result of the relationship between one melanocyte and 30-40 associated keratinocytes [12]. The ratio of melanocytes to keratinocytes is $1: 10$ in the epidermal basal layer (Figure 1). This balance is maintained through the human live but the exact mechanism is unknown [13]. About 1200 melanocytes exist per $\mathrm{mm}^{2}$ of the skin independently of the human race [14]. Adhesion molecules such as E- and P-cadherins participate in building cell-cell contact structures [15]. The contact between the dendritic processes of differentiated melanocyte and keratinocytes is necessary for the melanin transfer into keratinocytes determining the skin color and is involved in the skin cells' photoprotection. Melanin granules are accumulated above the keratinocyte nucleus and are removed with the shed epidermal cells [1]. The molecular mechanisms of the melanosomes transfer from melanocyte to keratinocytes is still a subject of investigations. Recently, Ando et al. proposed a model of melanosomes' transport via the shedding vesicle system through the following stages $[16,17]$ :

- surrounded by the membrane pigment globules (PG) containing multiple melanosomes and a few mitochondria are formed in the filopodia of melanocyte dendrites,

- PG are released from different areas of the dendrites into extracellular space,

- PG are captured by microvilli of keratinocytes, which incorporate them in a protease-activated receptor-2 (PAR-2)dependent way,

- membrane-surrounded PG is degraded,

- single melanosomes are released in a keratinocyte cytosol and reach the perinuclear area.

The size of the epidermal melanin units is similar independently of the human race, but varies between body areas. Racial distinctions are manifested in an arrangement of the melanocytic dendrites and in the melanogenesis intensity [18].

The precise mechanisms that control the organization and number of melanocytes in the epidermis are unknown. Melanocytes, keratinocytes and dermal fibroblasts communicate with each other by secreted factors and by cellcell contacts [19]. Keratinocytes control melanocyte growth and activity through a system of paracrine growth factors and cell adhesion molecules [13, 20]. Melanocytes and keratinocytes are also the local source of the different hormones which regulate melanocyte proliferation, melanogenesis and melanocytic dendrites' formation [21]. The cross-talking of different signaling pathways between keratinocytes and melanocytes is a part of an epidermal complex network involved in the maintenance of skin homeostasis. The basic elements of keratinocytes' influence on melanocytes' biology are illustrated in Figure 2 (based on [22-24]). In vitro, under ultraviolet radiation (UVR) keratinocytes increase the secretion of factors that influence many of melanocytes' biological activities summarized in Table 1 (based on $[24,25]$ ).

Melanocytes' biology is controlled also by dermal fibroblasts secreted factors, e.g. stem cell factor (SCF), neuregulin 1 (NRG1) $[23,26]$. These cytokines influence not only the growth and pigmentation of melanocytes, but also their shape, dendricity, mobility and adhesive properties [23, 27]. In the epidermal melanin unit melanocyte is a very active element that secrets a number of signal molecules targeting not only keratinocytes but also skin immunological system cells $[28,29]$. The proinflammatory cytokines (IL-1 $\alpha, \mathrm{IL}-2$, IL-3, IL-6, IL-10 and TNF- $\alpha$ ), chemokines (IL-8, CCL2), transforming growth factor (TGF- $\beta$ ), catecholamines, eicosanoids, serotonin, melanocyte stimulating factor $(\alpha-M S H)$ and nitric oxide (NO) are included as factors released by stimulated melanocytes [28, 29]. Secreted substances act also as autocrine factors, e.g. IL-1, IL-6 and TNF- $\alpha$ inhibit melanogenesis while under the influence of

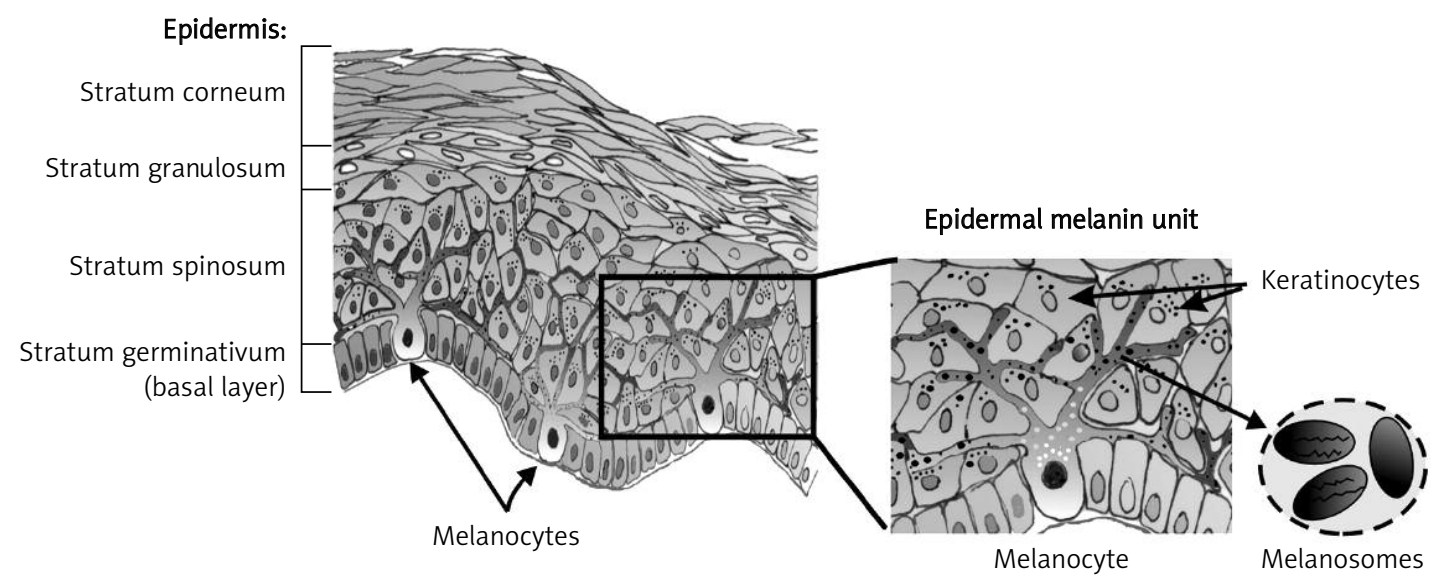

Figure 1. Scheme of the epidermis structure. Melanocyte reside between the basal layer cells and through dendritic processes communicates with about 30-40 keratinocytes in the epidermal melanin unit. Melanocyte synthesizes melanins in melanosomes transported into keratinocytes to protect them from UV radiation 


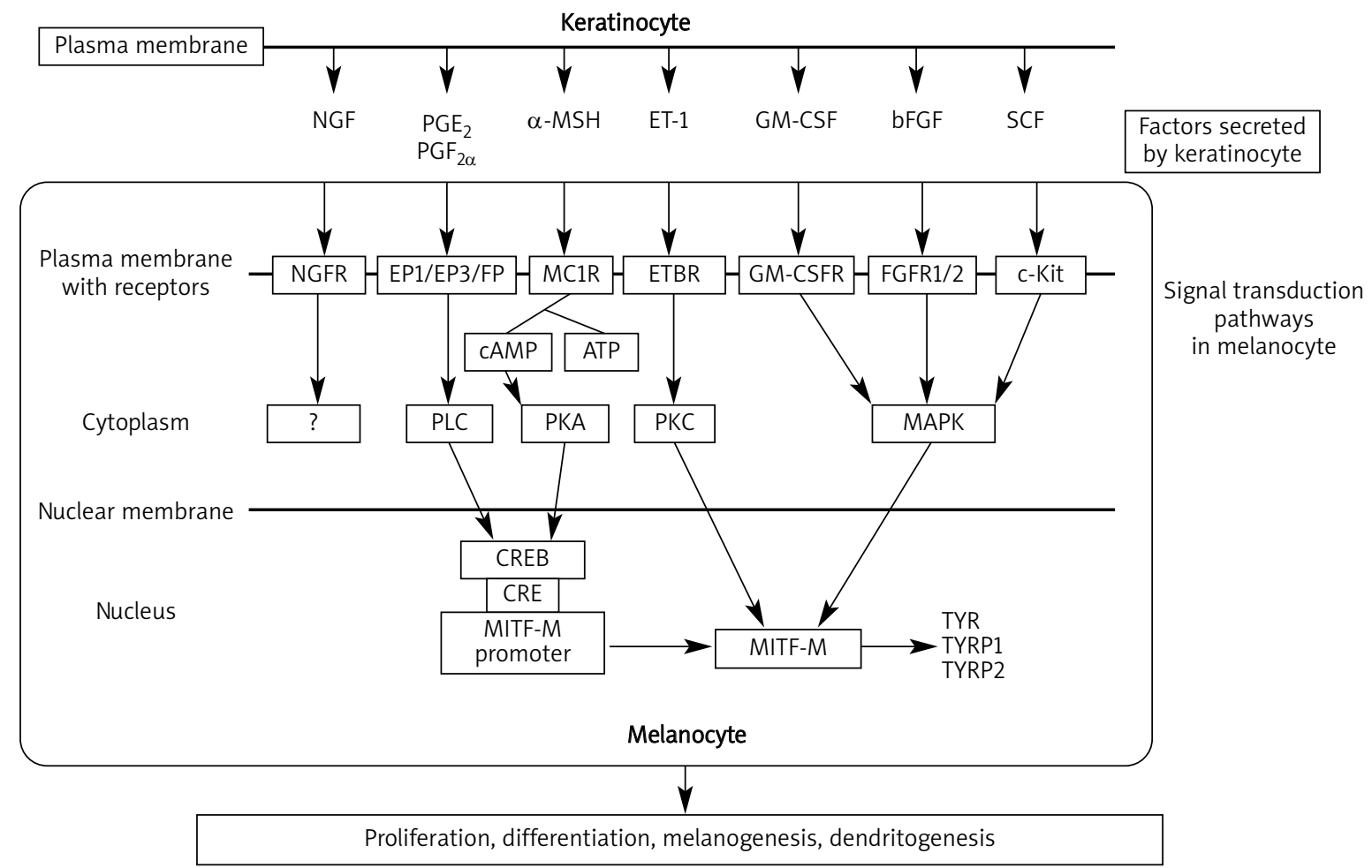

Figure 2. Graphical presentation of the basic elements in keratinocytes-melanocytes cooperation. The melanocyte proliferation, differentiation, melanogenesis are under control of factors secreted by surrounding keratinocytes SCF - stem cell factor, bFGF- basic fibroblast growth factor, GM-CSF - granulocyte-macrophage colony-stimulating factor, ET-1 - endothelin 1, $\alpha$-MSH - melanocyte-stimulating hormone, $P G E_{2}$ - prostaglandin $E_{2}, P G F_{2 \alpha}$ - prostaglandin $F_{2 \alpha}, N G F-$ nerve growth factor, c-Kit - tyrosine kinase receptor, FGFR1/2 - fibroblast growth factor receptor, GM-CSFR - granulocyte-macrophage colony-stimulating factor receptor, ETBR - endothelin B receptor, MC1R - melanocortin 1 receptor, EP1/EP3/FP - prostanoid receptors, NGFR - nerve growth factor receptor, MAPK - mitogen-activated protein (MAP) kinases, PKC - protein kinase C, PKA - protein kinase A, PLC - phospholipase C, TYR - tyrosinase, TYRP1 - tyrosinase-related protein 1, TYRP2 - tyrosinase-related protein 2, MITF-M - melanocyte-specific MITF (microphthalmia-associated transcription factor) isoform, CRE - CAMP response elements, CREB - CAMP response element-binding

Table 1. The paracrine factors secreted by keratinocytes after UV radiation that influence on melanocyte biology

\begin{tabular}{ll}
$\begin{array}{l}\text { The factors derived } \\
\text { from keratinocytes }\end{array}$ & The effects on melanocyte \\
\hline $\mathrm{bFGF}$ & $\uparrow$ Proliferation \\
\hline $\mathrm{ET}-1$ & $\begin{array}{l}\downarrow \text { Proliferation, } \uparrow \text { dendricity, } \\
\downarrow \text { melanogenesis }\end{array}$ \\
\hline $\mathrm{IL}-1 \alpha / 1 \beta$ & $\uparrow$ Proliferation, $\uparrow$ melanogenesis, \\
& $\uparrow$ survival \\
\hline $\mathrm{ACTH}$ & $\uparrow$ Proliferation, $\uparrow$ dendricity, \\
& $\uparrow$ melanogenesis, $\uparrow$ survival \\
\hline$\alpha-M S H$ & $\uparrow$ Dendricity, $\uparrow$ melanogenesis, \\
& $\uparrow$ melanosomal transfer \\
\hline $\mathrm{PGE} / \mathrm{PGF} \mathrm{F}_{2 \alpha}$ & $\uparrow$ Proliferation, $\uparrow$ melanogenesis \\
\hline $\mathrm{GM}-\mathrm{CSF}$ & $\uparrow$ Melanogenesis \\
\hline $\mathrm{NO}$ & $\downarrow$ Melanogenesis \\
\hline $\mathrm{TNF}-\alpha$ & $\uparrow$ Dendricity, $\uparrow$ survival \\
\hline $\mathrm{NGF}$ & $\downarrow$ Melanogenesis \\
\hline $\mathrm{BMP}-4$ & $\uparrow$ Proliferation, $\uparrow$ dendricity, \\
& $\uparrow$ melanogenesis \\
\hline
\end{tabular}

eicosanoids and $\alpha$-MSH the level of melanin synthesis is elevated [30]. Thus, melanocytes and cooperating keratinocytes form well-organized units in the epidermis. The stable element in each unit is the melanocyte that lives long, keratinocytes die and are shedding. It is an open question how long melanocyte lives in the human skin.

\section{Hair follicle melanocytes}

Melanocytes are located in the proximal bulb of each hair follicle and also near hair, e.g. in the sebaceous gland [31]. The bodies of bulbar melanocytes are located at the apex on the dermal papilla. Melanocyte dendrites enter between the cortical and medullar keratinocytes [32]. The ratio of melanocytes to keratinocytes is $1: 5$, it is more dense than in the epidermis (Figure 3) [33]. Follicular pigmentation is a result of structural and functional interactions between follicular melanocytes, matrix keratinocytes and dermal papilla fibroblasts. This tripartite system is described as the hair melanin unit or follicular melanin unit. The process of hair pigmentation includes the melanogenic activity of follicular melanocytes, the trans- 


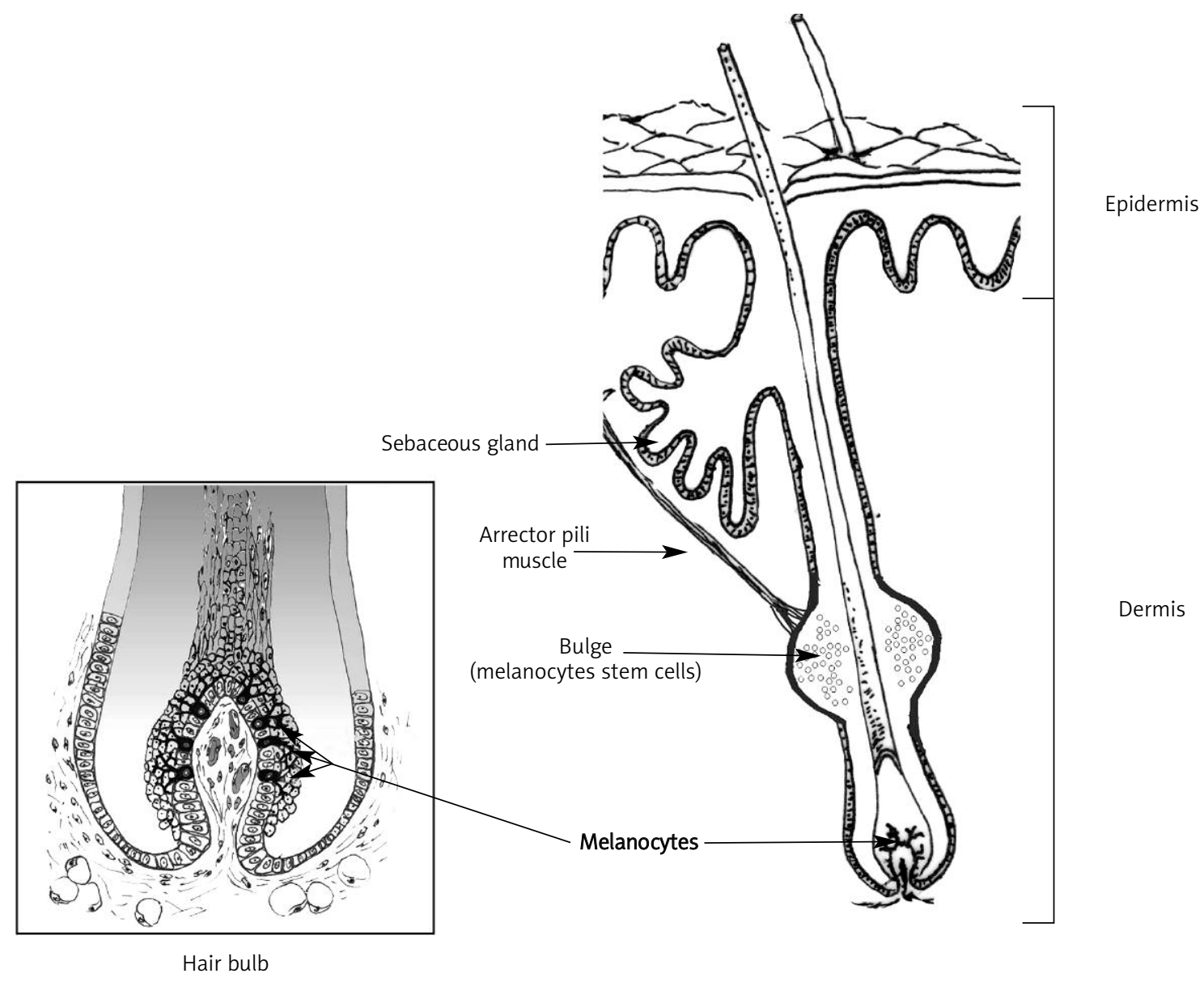

Figure 3. Melanocyte localization in the hair between cells covering the hair papilla in the hair bulb. Stem cells for melanocytes are located in the region named the hair bulge

fer of melanin granules into keratinocytes and the formation of pigmented hair shafts [32-34]. It is considered that a transport of melanin granules to keratinocytes in the growing hair shaft is similar to the epidermal phagocytosis of melanosomes mediated by receptor PAR2 on keratinocytes. But differences concern degradation of melanosomes and their quality. Hair melanocytes, in contrast to epidermal ones, include mainly bigger mature Stage IV melanosomes (melanogenesis and stages of melanosomes are described further in this work) and more expanded Golgi apparatus and rough endoplasmatic reticulum (RER). These pigment cells are larger and more dendritic than epidermal ones [31, 33]. Moreover, in epidermis almost whole transported melanin is degraded in the differentiating keratinocytes, but in hair cortical keratinocytes pigment granules are digested only slightly [33]. Diversity of hair color arises mostly from the quantity and ratio of the brown-black eumelanin and the yellow-red pheomelanin [35].

Melanin synthesis in the hair occurs under control of products secreted by neighboring cells as keratinocytes, fibroblasts and endothelial cells, which act through paracrine or autocrine mechanisms and may be modified by hormonal signals. In pigmentation determining hair color, the following elements are involved: melanocortin receptor 1 (MCR1) and its $\alpha$-MSH, adrenocorticotropic hormone (ACTH), receptor $\mathrm{C}-\mathrm{Kit}$ and its ligand SCF, endothelins, different neurotransmitters, cytokines, growth factor and other regulators similar as for epidermal melanogenesis control [31, 33]. The biochemical pathway of pigment formation and melanosomes biogenesis run likewise in the epidermis, but it is stressed that hair follicle melanocytes are more sensitive to aging influences than epidermal melanocytes, what results in hair greying [33]. It seems that fibroblast of dermal papilla derived factors: insulin growth factor (IGF-1), keratinocyte growth factor (KGF), noggin, SCF have special significance for control the hair matrix keratinocyte and melanocyte proliferation and activity during the hair growth $[32,36]$. Epidermal melanocytes are long-living cells, while hair melanocytes die at the end of the hair cycle which lasts 3-8 years [31]. The melanogenesis process takes place only during the anagen stage (growing phase) of the hair growth cycle; pigment formation is 
turned off in the catagen stage (regressing phase) and absent in the telogen stage (resting phase) [31]. Additionally, it is marked that during catagen completely differentiated bulbar melanocytes die through apoptosis, but new melanocytes develop from melanoblasts residing in the hair bulge [8, 32]. Summarizing, survival, proliferation and differentiation of melanocytes are regulated by microenvironment of the hair follicles.

\section{Melanocyte biology \\ Melanogenesis}

Melanogenesis is a biochemical pathway responsible for melanin synthesis [37]. It takes place in melanocytes, in separate cytoplasmic organelles called melanosomes [11]. Two major types of melanin are produced - pheomelanin and eumelanin. They differ in color and the way of synthesis. Melanin has numerous properties which are beneficial to the body: UV light absorption and scattering, free radical scavenging, coupled oxidation-reduction reactions and ion storage $[23,38,39]$. The availability of substrates and the function of melanogenesis enzymes decide about the types

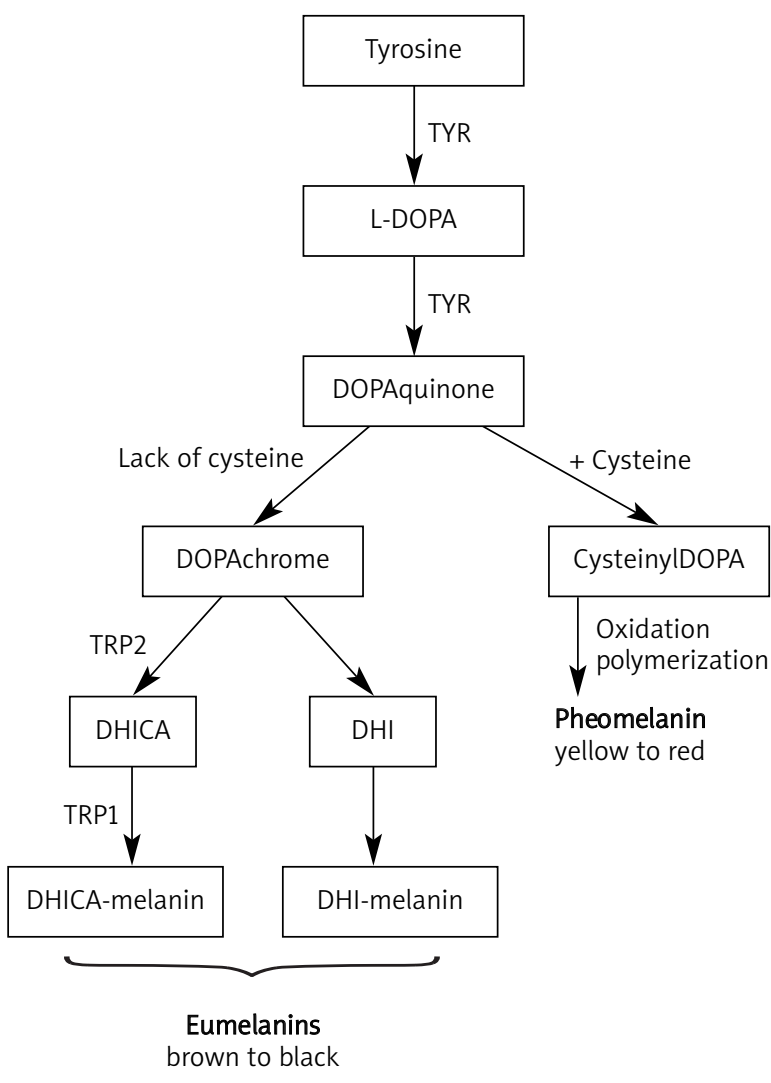

Figure 4. Simplified scheme of the melanin synthesis in melanocytes during melanogenesis. Tyrosine under influence of the basic enzymes such as tyrosinase (TYR), tyrosine-related protein 1 (TYRP1) and 2 (TYRP2) changes into a polymer of melanin, a mixture of pigments named eumelanin (black-brown) and pheomelanin (yellow-red) of melanins produced (Figure 4). Tyrosinase (TYR) carries out tyrosine hydroxylation to L-3,4-dihydroxyphenylalanine (DOPA) which is rapidly oxidized to DOPAquinone [40]. In the presence of cysteine DOPAquinone react with it, yielding 3- or 5-cysteinyIDOPAs, which then oxidize and polymerize, giving rise to yellow-red soluble melanin - pheomelanin $[37,41]$. In the absence of thiols (cysteine, glutathione or thioredoxin) brown-black eumelanin is produced. DOPAquinone spontaneously undergoes cyclization to DOPAchrome [42]. The DOPAchrome spontaneously loses carboxylic acid and generates 5,6-dihydroxyindole $(\mathrm{DHI})$, which rapidly oxidizes and polymerizes to form dark brown-black, insoluble DHI-melanin. However, if DOPAchrome tautomerase (TYRP2/DCT) is present, DOPAchrome will form DHI-2-carboxylic acid (DHICA) [43]. Tyrosinase and TYRP1 catalyze further conversions obtaining finally a lighter brown color DHICA-melanin [30, 37]. Human skin contains a mixture of all melanin types, and the ratio of those in part determines visible pigmentation [19]. Diversity of skin pigmentation among different ethnic groups is preserved and depends on eumelanin content. The ratio of eumelanin to total melanin decide about skin color [30]. Pheomelanin does not correlate with skin pigmentation, a similar amount of this pigment is observed in the dark and light skin. While in hair, the ratio of eumelanin to pheomelanin decides about the color [35]. Eumelanin comparing to pheomelanin has better photoprotecting properties - higher resistance to degradation and ability to reactive oxygen species (ROS) neutralization [44]. Eumelanins are considered to be more effective in terms of photoprotection than the reddish pheomelanin. As a consequence, the risk of skin cancer in lighter skin is 30-40-fold higher than in the darker one [41]. Products of genes regulating melanogenesis act at subcellular, cellular, tissue and environmental levels [21]. During melanogenesis, as intermediate products, cytotoxic molecules are synthesized (quinones, hydrogen peroxide). Thus, melanocyte protects itself by separating areas of melanogenesis in melanosomes and increases the level of antiapoptotic protein Bcl-2 [1, 21].

Melanosomes probably originate from endoplasmic reticulum of melanocytes, but it still remains a matter of debate [25]. Their development requires tyrosinase (TYR) and tyrosinase-related proteins (TYRP1, TYRP2). Of these three enzymes, tyrosinase is crucial to melanogenesis and is synthesized on the ribosomes of the RER and transported to the Golgi complex where it undergoes glycosylation, which is a process essential for its normal structure and function $[45,46]$.

There are four stages in melanosome development (Table 2). Premelanosomes (Stage I) are a round, small vesicles with an amorphous matrix. Melanosomes at Stage II have an organized, structured fibrillar matrix (mainly from gp100 family) and tyrosinase is present but pigment synthesis has not been noted. The beginning of melanin production takes place at Stage III, where pigment is deposited on protein fibrils. At the last Stage IV pigment fills the 
Table 2. Characteristics of the developmental stages of melanosomes during melanin synthesis. The melanogenesis takes place in special organelles named melanosomes. As first develops a vesicle (Stage I) which builds inside a fibrillar matrix formed by glycoproteins (Pmel17, MART-1) and gets tyrosinase and other enzymes of melanogenesis (Stage II). The melanosome produces melanin, which polymerizes and settles on the internal fibrils (Stage III). In the last stage (Stage IV) melanosome is filled up with melanin

\begin{tabular}{|c|c|c|c|c|}
\hline Melanosom features & Stage I & Stage II & Stage III & Stage IV \\
\hline Shape & Spherical & Elongated & Elliptical, ellipsoidal & Elliptical, ellipsoidal \\
\hline Internal structure & - & Matrix fibrils are visible & Matrix fibrils are visible & $\begin{array}{l}\text { Matrix fibrils are covered } \\
\text { by polymerized melanin }\end{array}$ \\
\hline TYR & - & + & + & + \\
\hline TYRP1 & - & + & + & + \\
\hline TYRP2 & - & + & + & + \\
\hline Melanin synthesis & - & - & Begins, settle on internal fibrils & Filled by melanin \\
\hline Color & & & Brown & Dark brown to black \\
\hline
\end{tabular}

whole melanosome [41, 47]. Fully melanized melanosomes lose tyrosinase activity and are transported to surrounding keratinocytes by elements of the cytoskeletal system (Figure 1) [48].

\section{Melanocytes' ability of proliferation and age-related changes}

The precise mechanisms that control the organization and number of melanocytes in the epidermis are unknown although keratinocytes may interact with melanocytes via growth factors, cell surface molecules, or other factors related to proliferation and differentiation of the epidermis. Melanocyte is a highly differentiated cell that produces a pigment melanin inside melanosomes. This cell is dark and dendritic in shape. Melanin production is the basic function of melanocyte. With the process of differentiation this cell loses the proliferative potential. Epidermal melanocytes are thought as a very stable population which proliferate extremely rarely under normal circumstances.

All we know about melanocytes' proliferation control come from in vitro studies. Human melanocyte proliferation requires the cross-talking of several signaling pathways including the MAPK-kinase signaling, $\alpha-M S H / C A M P / P K A$, Endothelin/PKC (PKA protein kinase A, PKC protein kinase C) $[23,49]$. As the potent mitogens are growth factors and hormones as stem cell factor (SCF), hepatocyte growth factor (HGF), endothelins, $\alpha-\mathrm{MSH}$, ACTH. While, transforming growth factor $-\beta$ (TGF- $\beta$ ), interferon- $\beta$ (INF- $\beta$ ), IL-1, IL-6, TNF- $\alpha$, cause the opposite effect and arrest melanocytes' growth [21, 29]. The MITF as the main melanocyte transcription factor influences proliferation, dendrite formation, melanin synthesis and induces the expression of antiapoptotic bcl-2 gene [50].

Epidermal melanocytes are long-living cells while hair melanocytes live as the hair cycle lasts (median: 3-5 years) [31]. Density of melanocytes in the skin depends on the environment (mainly UVR) and factors secreted by keratinocytes and fibroblasts. After 30 years of age $10-20 \%$ of epidermal melanocytes are lost every decade [51]. In the older people, apart from a decreased number of melanocytes morphology is changed (melanocytes are larger, more dendritic) and tyrosinase activity is reduced $[19,31,52]$. The relationships between ageing and the proliferative activity of melanocytes have been observed. In vitro, adult melanocytes proliferate less times than fetal and neonatal melanocytes [53]. Also, melanocytes from patients with a premature ageing disorder have reduced proliferative potential [50]. Terminally differentiated melanocyte proliferative potential is inhibited by changes in the cell cycle control elements, e.g. accumulation of cyclin-dependent kinase inhibitors (p27 Kip1, p16 INK4a and p21 Cip1), hypophosphorylation of $p R B$ (retinoblastoma protein), decrease level of cyclin D1 $[50,53]$. Table 3 lists basic cell cycle regulators involved in the regulation of melanocytic senescence (based on [53]).

Furthermore, the reason for a decreased number of melanocytes is programmed cell death of terminally dif- 
Table 3. The basic activity of the cell cycle regulators and other factors associated with melanocytes senescence

\begin{tabular}{ll}
\hline The cell cycle regulator & $\begin{array}{l}\text { The level of expression/ } \\
\text { activity during senescence }\end{array}$ \\
\hline Cyclin E & $\downarrow$ Protein level \\
\hline p16INK4a & $\uparrow$ Expression \\
\hline Protein RB1 & Dephosphorylation \\
\hline CDK2 and CDK4 & $\downarrow$ Activities \\
\hline Transcription factor E2F4 & $\uparrow$ Association with RB1 \\
\hline p300/CBP histone & $\downarrow$ Activity \\
acetyltransferases & $\uparrow$ Protein level \\
\hline Extracellular matrix proteins & Inactivation \\
\hline MAPK-signaling pathway & $\begin{array}{l}\text { Melanocytes senescence } \\
\text { is independent of p53, } \\
\text { probably except for } \\
\text { situation with deficient } \\
\text { p16/RB1 pathway }\end{array}$ \\
\hline p53 &
\end{tabular}

ferentiated cells. Accumulation of reactive oxygen substrates (ROS) as a result of reduced content/activity of catalase (key antioxidant enzyme) and downregulation of BCL-2 seem to be the main inducer of melanocyte apoptosis [50, 52].

In the melanocyte proliferation, the mitogen-activated protein kinase (MAPK) pathway is involved, which is stim- ulated by many growth factors. In the terminally differentiated melanocytes this main proliferative pathway is not active [50]. Discoveries in the field of molecular regulations of melanocyte proliferation and death help us understand disorders such as melanoma or vitiligo [54, 55].

Summarizing, the proliferation and differentiation of melanocytes during development are regulated by different genetic and epigenetic factors derived from keratinocytes, fibroblasts, melanocytes, the pituitary gland, other organs and environmental factors (such as UV radiation) [24].

\section{Embryonic origin of skin melanocytes \\ More than one population of neural crest cells is the source of skin melanocytes}

Neural Crest Cells is a group of cells originating from the embryonic germ layer named ectoderm. Under inductive influence of the notochord, the middle area of the embryonic disc differentiates into neuroectoderm that is visible as a neural plate at 4-week-old human embryo (Figure $5 \mathrm{~A}$ ). This plate folds and changes into the neural tube, future central nervous system elements - brain, spinal cord. During this process named neurulation, a group of cells from edges of the neural plate (crests), separates, changes the phenotype from epithelial to mesenchyme and migrate out from neuroepithelium (Figures $5 \mathrm{~A}$ and $5 \mathrm{~B}$ ). These neuroectodermal cells migrating to many places of the forming embryo's body are neural crest cells - NCC (Figure 5C). Neural crest cells are initially multipotent cells but gradually become lineage-restricted in developmen-
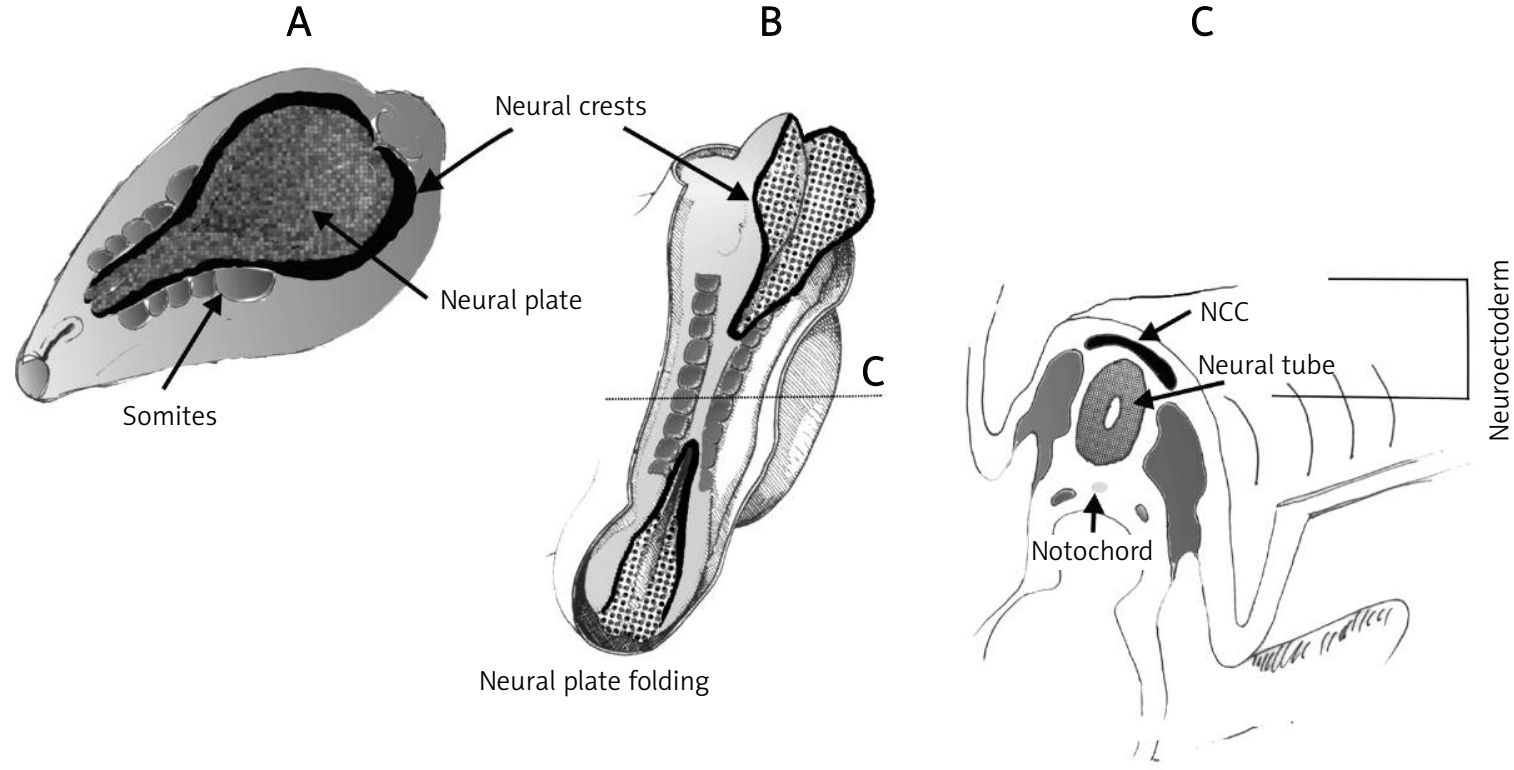

Figure 5. Development of the neural crest cells (NCC) during early embryogenesis at a 4-week-old embryo from neuroectoderm. The neuroectodermal cells proliferate, form the neural plate that folds, fuses and changes into neural tube (A, B). During this neurulation process, cells from edges (crests) of the neural plate separate from the neural tube as independent population of embryonic cells named neural crest cells, that is located above the neural tube (future brain and spinal cord) and beneath surface ectoderm (future epidermis) (C) 
tal potential. This potential is determined by anatomical localization along the cranial-caudal axis, e.g. cranial NCC can differentiate into neurons, glial cells but also chondrocytes, osteocytes, muscle cells, whereas trunk NCC form neurons and glial cells in the peripheral nervous system, endocrinal cells (Figure 6). These cells proliferate and start to express distinct molecular markers [7, 56].

Neural crest cells are traditionally grouped into four regionally distributed populations: cranial, vagal, trunk and sacral. Melanocytes mainly origin from cranial and trunklocated NCC.
Melanocytes residing in skin of the head origin from the cranial NCC while in the remaining parts of the human body mainly from the trunk NCC. Except melanocytes, cranial NCC together with mesodermal cells form the ectomesenchyme of the head, that gives skeleton, muscles and dermis of the head (Figure 6 B) [57].

According to embryonic migratory pathways, the trunk NCC is divided into two populations, dorsally (between surface ectoderm and somites) and ventrally (between neural tube and somites) migrating cells (Figure 6 A). Traditionally, the dorso-laterally migrating cells

\section{A Trunk NCC}

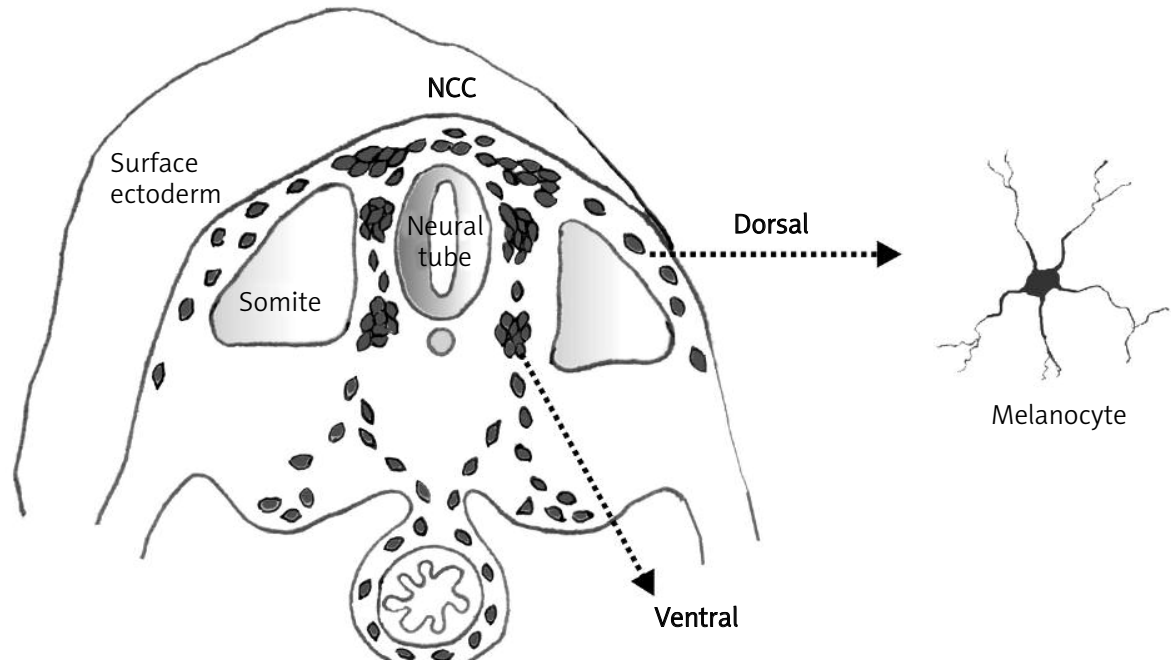

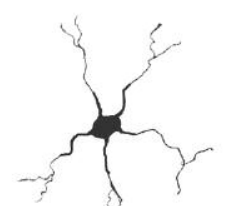

Melanocyte

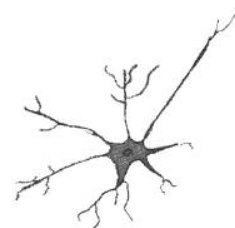

Neurons, glia of ganglia

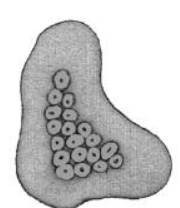

Adrenal medulla

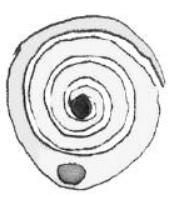

Schwann cells

B Cranial NCC

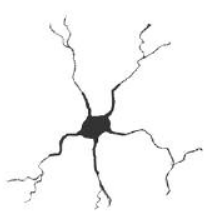

Melanocyte

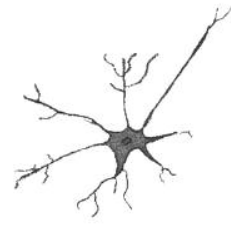

Neurons, glia of ganglia

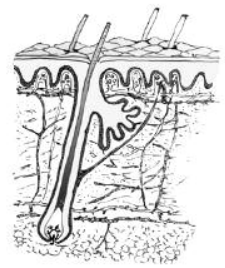

Dermis of head

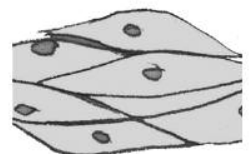

Smooth muscles

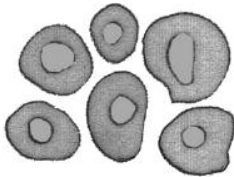

Chondrocyte osteocyte

Figure 6. Derivatives of the trunk (A) and cranial (B) neural crest cells (NCC) and the basic pathways of the trunk NCC migration during early embryonic time. Dorsally migrating trunk NCC move between the surface ectoderm and somites, finally develop into melanocytes of the epidermis and hair (A). Ventrally migrating trunk NCC move between the neural tube and somites, give elements of the peripheral nervous system (ganglionic cells, Schwann cells), medulla of the adrenal glands and according to latest investigations, melanocytes of the skin (A) 
are thought to be the main source for melanocytes while the ventrally migrating cells give rise to the peripheral nervous system and adrenal medulla (Figure 6 B). But, there is strong evidence that a fraction of melanocytes arise from cells migrating first ventrally and then along the nerves $[9,58]$. Cells present in a nerve sheath (Schwann cells) have the potential to produce melanocytes also after birth. In vitro, Schwann cells cultured in melanocytes medium de-differentiate into glial-melanocytic progenitor able to give melanocytes [59].Thus, cells migrating ventrally either differentiate into neurons or are maintained as multipotent cells that differentiate into cells forming myelin sheath or melanocytes (Figure 6) [7]. These cells invade the epidermis during the process of embryonic cutaneous innervation [9]. The recent findings that congenital (prenatal) nevi begin as intradermal nevi seems to support the hypothesis that precursors for melanocytes could origin from dermis cells [60]. It is suggested that prenatal nevi may develop from the precursors for Schwann cells, which arrive near epidermis along cutaneous nerve, may respond to factors secreted by epidermal cells and differentiate into melanocytes. As cutaneous nerves grow from deep dermis near the epidermis they branch and form the candelabra pattern (a neurocutaneous unit). Along these branches precursors for melanocytes migrate to the epidermis and as a result the congenital nevi may develop [60]. During human development, melanoblast migration and cutaneous nerve growth take place at the same time between 6 and 8 weeks $[9,60]$. Communication between the nervous system and epidermal melanocytes has been proven [61]. The observation that epidermal melanocytes molecularly differ from dermal melanocytes seems to support the hypothesis about double origin of skin melanocytes [62]. Thus, melanocytes in the skin either derive directly from NCC populating the skin via a dorsolateral migratory pathway or arise from ventrally migrating precursors forming the myelin around the cutaneous nerves [63].

As melanoblasts travel through the dermis, they multiply. While traveling to their final destinations, melanoblasts sequentially express additional melanogenic genes, many of them regulated by transcription factor MITF. The most important in the maturation of melanocyte is the appearance of tyrosinase, enzyme of melanin synthesis. Melanocytes finally reside in the skin and hair follicles, the oral mucosa, the choroid of the eye, the iris, and some internal sites, such as meninges and the inner ear (the stria vascularis). The fate of NCC depends on environmental factors they meet on the migratory pathways [7]. After cell specification, melanoblasts proliferate and spread to their final destinations in the epidermis and hair follicles where they differentiate. It takes place at 6-8 weeks and by $12-13$ weeks the majority are localized in the epidermis $[60,64]$. Whether all of them reach the epidermis is an unresolved developmental problem. Dermal melanocytes are seen during human fetal development but they are not evident after birth. There are suggestions that some melanoblasts could stay in the dermis $[64,65]$. It remains unknown how the stream of melanoblasts to the epidermis is controlled. The time when melanoblast presence in the dermis is detectable is also time for cutaneous nerve development [60]. The time between 9 and 12 weeks is also the beginning of hair buds' development and melanoblast migration to them. On the $18^{\text {th }}$ week of intrauterine life a hair which comes through the skin surface have melanocytes present in the hair bulb $[64,66]$ but the activity of tyrosinase is very low [67]. The fetal first hair present on the whole embryo skin is named lanugo. At the end of pregnancy (30-33 weeks) lanugo degenerates and final hair develops, the first hair cycle is done [68].

Spaces between cells are filled with a rich extracellular matrix formed by many fibrillar proteins such as collagens, fibronectin. Cell adhere to one another using adhesion molecules such as cadherins or adhere to matrix by integrins. These adhesion mechanisms are of special importance during melanoblast migration. Melanoblasts migrate over very long distances throughout the embryo, proliferate and promote their own survival. Thus, melanoblast development is a highly dynamic process, which requires rapid activation of different signaling pathways.

\section{Basic molecular elements of embryonic/ fetal melanocytes development}

The results of experiments have not found out an answer to a question - at what stage NCC get features of melanoblasts, precursors to melanocytes [7, 69]. Melanoblasts have features of melanocytes but do not produce melanin, there is no agreement about a set of molecular markers for this early stage of melanocyte in human development. The most commonly listed molecular markers for the precursors for melanocytes are: a tyrosine kinase receptor KIT (c-kit); transcription factors such as MITF, SOX10, Pax 3 and melanogenic enzyme tyrosinase-related protein (TYRP-2) [7, 24, 56, 70]. The exact mechanisms responsible for melanoblast migration are not well understood, although adhesion molecules such as cadherins, integrins, and extracellular matrix elements are involved in it. The ephrin receptor (EphR) and the endothelin receptor (EDNRB2) allow melanoblasts to migrate along extracellular matrix containing ephrin and endothelin-3 [69, 71].

The most important growth factors regulating melanocyte development from the NCC are endothelins, ligand for c-kit (SCF stem cell factor), Wnt proteins and neuregulin-1 $[72,73]$. This regulations are described in detail in other works $[24,63,72,73]$.

The c-Kit receptor binds the stem cell factor (SCF) secreted by the dermal cells and as a result of this pathway activation melanoblasts avoid apoptosis and proliferate [24, 73]. The Wnt/Frizzled protein/ $\beta$-catenin-signaling pathway, the Notch pathway and the MAPK-signaling pathways are essential for melanoblast/melanocyte development. Studies in several model organisms suggest that compo- 
nents of the $W n t / \beta$-catenin signaling pathway are required for induction of melanocyte fate [74]. The Notch signaling pathway is of special importance during the embryonic period. The Notch receptor requires the contact with another cell because the ligand for this receptor is a plasma membrane protein (e.g. Delta). MAPK-signaling pathway Ras/Raf/Mek/Erk) has been of special interest according to BRAF role in melanoma development [75].

Many of the factors listed above induce expression of the MITF protein functioning as a master of melanocyte proliferation, differentiation and survival. The MITF activates genes responsible for the migration to the skin, preventing apoptosis in migrating cells, melanin production [70, 76].

\section{Stem cells for human melanocytes in adults}

Melanocytes proliferate rarely but some situations point out that in the skin there are precursors for melanocytes e.g. repigmentation of the skin of vitiligo patients after phototherapy [77].

What do we know about the presence of melanoblasts in the adult skin?

The first documented reservoir for melanocyte stem cells was the bulge area of the hair follicles either in the mouse or human $[78,79]$. The bulge area is a part of outer root sheath that provides the insertion point for the arrector pili muscle and points to the bottom of the permanent portion of hair follicles (Figure 3). Each time a hair is lost the hair follicle regenerates and melanocyte stem cells are activated [80]. The bulge region contains pluripotential, morphologically undifferentiated cells which develop into keratinocyte progenitors and melanoblasts not only for hair but also epidermis [8]. After stimulation these cells migrate in the basal layer of the epidermis and differentiate into mature melanocytes $[64,78]$.

However, there is growing evidence that the dermis is also a reservoir of melanocytes [64, 77, 81]. Majority of the progress on melanocyte development has been made in murine models, little progress has been made on the identification of melanocyte-producing stem cells in human skin. Where are melanoblasts located in the dermis? The answer to this question is not satisfactory enough. Recent developmental studies using model organisms and lineage tracing have been able to trace melanocytes arising from migration of a multipotent precursor cell along nerve projections. With great probability there could be cells with stem cell's properties located in the cutaneous nerves, but if only $[9,58]$ ? These cells are retained in a stem cell-like state until the signal sent by the end of the cutaneous nerve promotes these cells to differentiate into melanocytes [82]. But there are also observations that some melanoblasts from the dorsal way of NCC migration stay in the dermis after the end of epidermis inoculation, up to the second trimester during fetal time $[64,71,83]$. Whether they survive in the human adult dermis is still an open problem.
There are difficulties in the identification of melanoblasts in the adult skin because of lack of a set of melanoblast markers. It seems that the epidermis and dermis melanocytes are biologically different populations [62].

Epidermis and dermis as places for the melanoblasts' reservoir give an opportunity for a new look at the melanoma heterogeneity. Melanoma is a tumor which develops from melanocytes but there is no agreement if the mature melanocytes or cells from earlier developmental stages may follow the tumoricidal transformation [65, 8486]. Recently, Whiteman et al. made an attempt to separate melanoma(s) into melanoma arising from epithelial melanocytes and not associated with epithelia [86].

\section{References}

1. Plonka PM, Passeron T, Brenner DJ, et al. What are melanocytes really doing all day long...? Exp Dermatol 2009; 18: 799-819.

2. Tachibana M. Sound needs sound melanocytes to be heard. Pigment Cell Res 1999; 12: 344-54.

3. Brito FC, Kos L. Timeline and distribution of melanocyte precursors in the mouse heart. Pigment Cell Melanoma Res 2008; 21: 464-70.

4. Hu DN, Simon J, Sarna T. Role of ocular melanin in ophthalmic physiology and pathology. Photochem Photobiol 2008; 84: 639-44.

5. Randhawa M, Huff T, Valencia JC, et al. Evidence for the ectopic synthesis of melanin in human adipose tissue. FASEB J 2009; 23: 835-43.

6. O'Rahilly R, Müller F. The development of the neural crest in the human. J Anat 2007; 211: 335-51.

7. Ernfors P. Cellular origin and developmental mechanisms during the formation of skin melanocytes. Exp Cell Res 2010; 316: 1397-407.

8. Nishimura EK. Melanocyte stem cells: a melanocyte reservoir in hair follicles for hair and skin pigmentation. Pigment Cell Melanoma Res 2011; 24: 401-4.

9. Adameyko I, Lallemend F, Aquino JB, et al. Schwann cell precursors from nerve innervation are a cellular origin of melanocytes in skin. Cell 2009; 139: 366-79.

10. Cramer SF. Stem cells for epidermal melanocytes - a challenge for students of dermatology. Am I Dermatopathol 2009; 31: 331-431.

11. Seiji H, Fitzpatrick TB. The reciprocal relationship between melanization and tyrosinase activity in melanosomes (melanin granules). J Biochem 1961; 49: 700-6.

12. Fitzpatrick TB, Breathnach AS. The epidermal melanin unit system. Dermatol Wochenschr 1963; 147: 481-9.

13. Haass NK, Herlyn M. Normal human melanocyte homeostasis as a paradigm for understanding melanoma. J Invest Dermatol Symp Proc 2005; 10: 153-63.

14. Miot LD, Miot HA, Silva MG, et al. Physiopathology of melasma. An Bras Dermatol 2009; 84: 623-35.

15. Haass NK, Smalley KS, Li L, et al. Adhesion, migration and communications in melanocytes and melanoma. Pigment Cell Res 2005; 18: 150-9.

16. Ando H, Niki Y, Yoshida M, et al. Involvement of pigment globules containing multiple melanososmes in the transfer of melanosomes from melanocytes to keratinocytes. Cell Logist 2011; 1: 12-20.

17. Ando H, Niki Y, Masaaki I, et al. Melanosomes are transferred from melanocytes to keratinocytes through the processes of 
packing, release, uptake, and dispersion. J Invest Dermatol 2012; 132: $1222-9$.

18. Miyamura Y, Coelho S, Wolber R. Regulation of human skin pig mentation and responses to ultraviolet radiation. Pigment Cell Res 2006; 20: 2-13.

19. Yamaguchi Y, Brenner M, Hearing VJ. The regulations of skin pigmentation. J Biol Chem 2007; 13: 1-11.

20. Lee AY. Role of keratinocytes in the development of vitiligo. Ann Dermatol 2012; 24: 115-25.

21. Sulaimon SS, Kitchell BE. The biology of melanocytes. Vet Dermatol 2003; 14: 57-65.

22. Hirobe T. Role of keratinocyte-derived factors involved in regulating the proliferation and differentiation of mammalian epidermal melanocytes. Pigment Cell Res 2004; 18: 2-12.

23. Costin GE, Hearing VJ. Human skin pigmentation: melanocytes modulate skin colour in response to stress. FASEB J 2007; 21: 976-94.

24. Hirobe T. How are proliferation and differentiation of melanocytes regulated? Pigment Cell Melanoma Res 2011; 24: 462-78.

25. Park HY, Kasmadaki M, Gilchrest YBA. Cellular mechanisms regulating melanogenesis. Cell Mol Life Sci 2009; 66: 1493-506.

26. Choi W, Kolbe L, Hearing VJ. Characterization of the bioactive motif of neuregulin-1, a fibroblast-derived paracrine factor that regulates the constitutive color and the function of melanocytes in human skin. Pigment Cell Melanoma Res 2012; 25: 1-5.

27. Wang Z, Coleman DJ, Bajaj G, et al. RXRalpha ablation in epidermal keratinocytes enhances UVR-induced DNA damage, apoptosis, and proliferation of keratinocytes and melanocytes. J Invest Dermatol 2011; 131: 177-87.

28. Lu Y, Zhu WY, Tan C, et al. Melanocytes are potential immunocompetent cells: evidence from recognition of immunological characteristics of cultured human melanocytes. Pigment Cell Res 2002; 15: 454-60.

29. Tam I, Stępień K. Melanocytes - immunocompetent pigment cells. Postep Derm Alergol 2007; 4: 188-93.

30. Slominski A, Tobin DJ, Shibahara S, et al. Melanin pigmentation in mammalian skin and its hormonal regulation. Physiol Rev 2004; 84: 1155-228.

31. Tobin DJ. The cell biology of human hair follicle pigmentation. Pigment Cell Melanoma Res 2011; 24: 75-88.

32. Commo S, Bernard BA. Melanocyte subpopulation turnover during the human hair cycle: an immunohistochemical study. Pigment Cell Res 2000; 13: 253-9.

33. Slominski A, Wortsman J, Plonka PM, et al. Hair follicle pig mentation. J Invest Dermatol 2005; 124: 13-21.

34. Commo S, Gaillard O, Thibaut S, et al. Absence of TRP-2 in melanogenic melanocytes of human hair. Pigment Cell Res 2004; 17: 488-97.

35. Ito S, Wakamatsu K. Diversity of human hair pigmentation as studied by chemical analysis of eumelanin and pheomelanin. J Eur Acad Dermatol Venereol 2011; 25: 1369-80.

36. Randall VA. Androgens and hair growth. Dermatol Ther 2008; 21: 314-28.

37. Simon DJ, Peles D, Wakamatsu K, et al. Current challenges in understanding melanogenesis: bridging chemistry, biological control, morphology and function. Pigment Cell Melanoma Res 2009; 22: 563-79.

38. Riley PA. Melanin. Int J Biochem Cell Biol 1997; 29: 1235-9.

39. Bush WD, Simon JD. Quantification of $\mathrm{Ca}(2+)$ binding to melanin supports the hypothesis that melanosomes serve a functional role in regulating calcium homeostasis. Pigment Cell Res 2007; 20: 134-9.
40. Fitzpatrick TB, Miyamoto M, Ishikawa K. The evolution of concepts of melanin biology. Arch Dermatol 1967; 96: 305-23.

41. Hearing VJ. Determination of melanin synthetic pathway. J Invest Dermatol 2011; 131: 8-11.

42. Sugumaran M. Molecular mechanism for mammalian melanogenesis. Comparison with insect cuticular sclerotization. FEBS Lett 1991; 295: 233-9.

43. Del Marmol V, Beerman F. Tyrosinase and related proteins in mammalian pigmentation. FEBS Lett 1996; 381: 165-8.

44. Abdel-Malek ZA, Kadekaro AL, Swope VB. Stepping up melanocytes to the challenge of UV exposure. Pigment Cell Melanoma Res 2010; 23: 171-86.

45. Hearing VJ. Biogenesis of pigment granules: a sensitive way to regulate melanocyte function. J Dermatol Sci 2005; 37: 3-14.

46. Watabe H, Valencia JC, Le Pape E, et al. Involvement of dynein and spectrin with early melanosome transport and melanosomal protein trafficking. J Invest Dermatol 2008; 128: 162-73.

47. Schiaffino MV. Signaling pathways in melanosome biogenesis and pathology. Int J Biochem Cell Biol 2010; 42: 1094-104.

48. Schallreuter K, Slominski A, Pawelek JM. What controls melanogenesis? Exp Dermatol 1998; 7: 143-50.

49. Mizoguchi M. Melanocyte development: with a messenger of encouragement to young women scientists. Pigment Cell Res 2004; 17: 533-544.

50. Rizos H, Becker TM, Holland EA. Cell cycle regulation in the melanocyte. In: Textbook of melanoma. Thomson JF, Morton DL, Kroon BBR (eds.). Martin Dunitz Taylor \& Francis Group UK, London 2004; 100: 13-25.

51. Whiteman DC, Parsons PG, Green AC. Determinants of melanocyte density in adult human skin. Arch Dermatol Res 1999; 291: 511-6.

52. Kauser S, Westgate GE, Green MR, et al. Human hair follicle and epidermal melanocytes exhibit striking differences in their aging profile which involves catalase. I Invest Dermatol 2011; 131: 979-82.

53. Bennet DC, Medrano EE. Molecular regulation of melanocyte senescence. Pigment Cell Res 2002; 15: 242-50.

54. Imokawa G. Autocrine and paracrine regulation of melanocytes in human skin and in pigmentary disorders. Pigment Cell Res 2004; 17: 96-110.

55. Misterska M, Szulczynska-Gabor J, Zaba R. Aetiopathogenesis, clinical picture and treatment of vitiligo. Postep Derm Alergol 2009; 26: 212-23.

56. Betters E, Liu Y, Kjaeldgaard A, et al. Analysis of early human neural crest development. Dev Biol 2010; 344: 578-92.

57. Le Douarin NM, Creuzet S, Couly G, et al. Neural crest cell plasticity and its limits. Development 2004; 131: 4637-50.

58. Cramer SF. The histogenesis of acquired melanocytic nevi-based on a new concept of melanocytic differentiation. Am J Dermatopathol 1984; 6: 289-98.

59. Dupin E, Real C, Glavieux-Pardanaud C, et al. Reversal of developmental restrictions in neural crest cells lineages: transition from Schwann cells to glial-melanocytic precursors in vitro. Proc Natl Acad Sci USA 2003; 100: 5229-33.

60. Cramer SF, Fesyuk A. On the development of neurocutaneous units-implications for the histogenesis of congenital, acquired, and dysplastic nevi. Am J Dermatopathol 2012; 34: 60-81.

61. Hara M, Toyoda M, Yaar M, et al. Innervation of melanocytes in human skin. J Exp Med 1996; 184: 1385-95.

62. Aoki H, Yamada Y, Hara A, et al. Two distinct types of mouse melanocyte: differential signaling requirement for the maintenance of non-cutaneous and dermal versus epidermal melanocytes. Development 2009; 136: 2511-21. 
63. Sommer L. Generation of melanocytes from neural crest cells. Pigment Cell Melanoma Res 2011; 24: 411-21.

64. Gleason BC, Crum CP, Murphy GF. Expression patterns of MITF during human cutaneous embryogenesis: evidence for bulge epithelial expression and persistence of dermal melanoblasts. J Cutan Pathol 2008; 35: 615-22.

65. Zabierowski SE, Fukunaga-Kalabis M, Li L, et al. Dermis-derived stem cells: a source of epidermal melanocytes and melanoma? Pigment Cell Melanoma Res 2011; 24: 422-9.

66. Muller M, Jasmin JR, Monteil RA, Loubiere R. Embryology of the hair follicle. Early Hum Dev 1991; 26: 159-66.

67. Gerschoni-Baruch R, Benderly A, Brandes JM, et al. DOPA reaction test in hair bulbs of fetuses and its application to the prenatal diagnosis albinism. J Am Dermatol 1991; 24: 220-2.

68. Gareri J, Koren G. Prenatal hair development : implications for drug exposure determination. Forensic Science International 2010; 196: 27-31.

69. Harris ML, Erickson CA. Lineage specification in neural crest cell path finding. Dev Dyn 2007; 236: 1-19.

70. Vance KW, Goding CR. The transcription network regulating melanocyte development and melanoma. Pigment Cell Res 2004; 17: 318-25.

71. Dupin E, Sommer L. Neural crest progenitors and stem cells: from early development to adulthood. Dev Biol 2012; 366: 83-95.

72. Cooper CD, Raible DW. Mechanisms for reaching the differentiated state: insights from neural crest-derived melanocytes. Semin Cell Dev Biol 2009; 20: 105-10.

73. Hari L, Miescher I, Shakhova O, et al. Temporal control of neural crest lineage generation by Wnt/beta-catenin signaling. Development 2012; 139: 2107-17.

74. Raible DW, Ragland JW. Reiterated Wnt and BMP signals in neural crest development. Semin Cell Dev Biol 2005; 16: 673-82.

75. Smalley KS. A pivotal role for ERK in the oncogenic behavior of malignant melanoma? Int J Cancer 2003; 104: 527-32.

76. McGill GG, Horstmann M, Widlund HR, et al. Bcl2 regulation by the melanocyte master regulator Mitf modulates lineage survival and melanoma cell viability. Cell 2002; 109: 707-18.

77. Davids LM, du Toit E, Kidson SH, et al. A rare repigmentation pattern in a vitiligo patient: a clue to an epidermal stem-cell reservoir of melanocytes? Clin Exp Dermatol 2009; 34 246-8.

78. Nishimura E, Jordan SA, Oshima $\mathrm{H}$, et al. Dominant role of the niche in melanocyte stem-cell fate determination. Nature 2002; 416: 854-60.

79. Nishimura EK, Granter SR, Fisher DE. Mechanisms of hair graying: incomplete melanocyte stem cell maintenance in the niche. Science 2005; 307: 720-4.

80. Goding CR. Melanocytes, the new black. Int J Biochem Cell Biol 2007; 39: 275-9.

81. Toma JG, McKenzie IA, Bagli D, et al. Isolation and characterization of multipotent skin-derived precursors from human skin. Stem Cells 2005; 23: 727-37.

82. Adameyko I, Lallemend F, Furlan A, et al. Sox2 and Mitf crossregulatory interactions consolidate progenitor and melanocyte lineages in the cranial neural crest. Development 2012; 139: 397-410.

83. Suder E, Bruzewicz S. Melanocytes of fetal dermis - studies with anti-HMB-45 antibody. Med Sci Monit 2004; 10: 229-32.

84. Schatton T, Frank MH. Cancer stem cells and human malignant melanoma. Pigment Cell Melanoma Res 2007; 21: 39-55.

85. Hoek KS, Goding CR. Cancer stem cells versus phenotypeswitching in melanoma. Pigment Cell Melanoma Res 2010; 23: 746-59.
86. Whiteman DC, Pavan WJ, Bastian BC. The melanomas: a synthesis of epidemiological, clinical, histopathological, genetic, and biological aspects, supporting distinct subtypes, casual pathways, and cells of origin. Pigment Cell Melanoma Res 2011; 24: 879-97. 